Provided for non-commercial research and education use. Not for reproduction, distribution or commercial use.

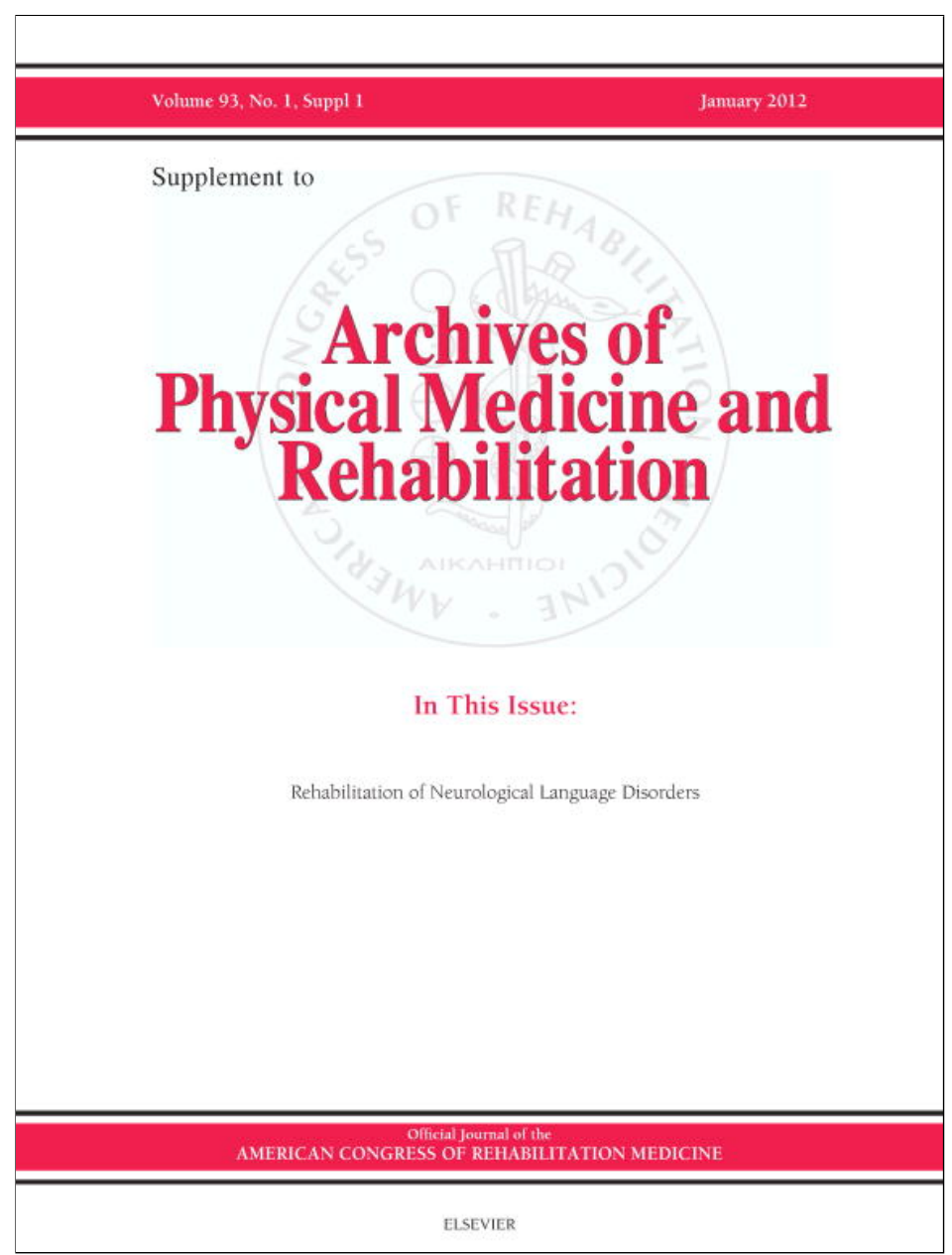

This article appeared in a journal published by Elsevier. The attached copy is furnished to the author for internal non-commercial research and education use, including for instruction at the authors institution and sharing with colleagues.

Other uses, including reproduction and distribution, or selling or licensing copies, or posting to personal, institutional or third party websites are prohibited.

In most cases authors are permitted to post their version of the article (e.g. in Word or Tex form) to their personal website or institutional repository. Authors requiring further information regarding Elsevier's archiving and manuscript policies are encouraged to visit:

http://www.elsevier.com/copyright 


\title{
Using Phonemic Cueing of Spontaneous Naming to Predict Item Responsiveness to Therapy for Anomia in Aphasia
}

\author{
Paul J. Conroy, PhD, Claerwen Snell, PhD, Karen E. Sage, PhD, Matthew A. Lambon Ralph, PhD
}

ABSTRACT. Conroy PJ, Snell C, Sage KE, Lambon Ralph MA. Using phonemic cueing of spontaneous naming to predict item responsiveness to therapy for anomia in aphasia. Arch Phys Med Rehabil 2012;93(1 Suppl 1):S53-60.

Background: Anomia refers to difficulties retrieving words and is 1 of the most common symptoms of aphasia and hence often the target of therapy. The principal aim of the present study was to explore, for the first time, whether it is possible to predict the responsiveness of individual words to naming therapy from the psycholinguistic properties of those words and from the length of the phonemic cue required to name them. The relationship between this form of cueing and the outcome of naming therapy is of particular interest given that cueing is an established research and clinical tool within aphasiology, and is commonly used to probe naming performance.

Method: By amalgamating data from 3 previous studies, we were able to analyze data from 22 participants with chronic aphasia, yielding cueing and therapy data for 1080 target words. Cross-session changes in cueing and naming accuracy were collated for 298 target words.

Results: The results demonstrated that items which were accurately named after therapy (both at $1 \mathrm{wk}$ and $5 \mathrm{wk}$ later) required a significantly shorter phonemic cue to prompt correct naming in assessments prior to therapy. Imageability was a significant predictor of the required cue level, whereas word age of acquisition and word frequency were not. Highly imageable words required less cueing and were more likely to be accurately named posttherapy. A novel analysis of cross-session accuracy revealed that, even though the required cue length reduced across the first 6 of 10 therapy sessions, the relationship between the required cue length and final posttherapy accuracy was present throughout therapy.

Discussion: The findings are discussed in the context of their clinical implications for intervention, specifically for therapies that focus on accurate production of specific word targets. Themes for future related research are also considered.

Key Words: Aphasia; Anomia; Rehabilitation; Stroke; Therapy.

(C) 2012 by the American Congress of Rehabilitation Medicine

From the Neuroscience and Aphasia Research Unit, University of Manchester, Manchester (Conroy, Sage, Lambon Ralph); and Warrington National Health Service, Warrington (Snell), UK

Supported by a research bursary from the UK Stroke Association (no. TSAB 2004/01); and the Medical Research Council.

No commercial party having a direct financial interest in the results of the research supporting this article has or will confer a benefit on the authors or on any organization with which the authors are associated.

Reprint requests to Paul Conroy, PhD, Neuroscience and Aphasia Research Unit, Zochonis Building, University of Manchester, Manchester M13 9PL, UK, e-mail: Paul.Conroy@manchester.ac.uk.

0003-9993/12/9301S-00996\$36.00/0

doi:10.1016/j.apmr.2011.07.205
CUEING HAS A LONG tradition in aphasiology. ${ }^{1,2}$ From a therapeutic point of view, cueing makes intuitive sense. Using physiotherapy as an analogy, a physiotherapist may support an activity, such as walking, through as little manual help as possible; minimal support has an immediate logic given the therapeutic need to reduce that support as soon as possible to ensure independent walking. Similarly, within the domain of aphasia and particularly in relation to therapy for anomia, cueing typically involves the clinician or researcher providing minimal prompts designed to aid the person with aphasia in a testing or therapy task (classically, picture naming) and then offering more substantial prompts if the task has not been achieved successfully. In confrontation naming, cues tend to be either phonemically based (eg, a verbal cue consisting of the first consonant or first consonant plus vowel in a target word), graphemically based (a letter cue, eg, first grapheme(s) of a target word), both phonemic and graphemic, or use sentence completion or word/sentence repetition. ${ }^{3}$

A distinction made in the early cognitive neuropsychologic literature was between facilitation, when a cue is used on 1 occasion, and treatment, when a cue is used repeatedly in order to achieve more lasting effects. ${ }^{4}$ Initially, treatment using semantic/word meaning cues (eg, word definitions, word-to-picture matching, semantic judgements, categorizing according to meaning) was thought to be more effective than treatment using phonologic/word sound cues. ${ }^{5}$ However, the current consensus has been that the outcome of picture naming therapies based on semantic or phonologic cues are indistinguishable, given that word phonology is activated in semantic tasks and semantics is activated in phonologic tasks, especially given that the target picture will tend to activate the target semantics. ${ }^{6}$

Responsiveness to cues in picture naming assessment might provide a positive prognostic indicator for treatment. ${ }^{7}$ Hickin et $\mathrm{al},{ }^{5}$ in a case-series study of 8 participants with aphasia undergoing naming therapy using phonologic and orthographic cues, found a significant correlation between the effect of cue facilitation and overall therapy outcome for the group as a whole. Referring to the initial degree of responsiveness to cueing as facilitation, participants were presented with the picture of an object representing a short word (consisting of a consonantvowel-consonant only, eg, pen) to name, and if unable to do so were given 1 of 3 types of cues. These were a single cue, a choice cue, or the control condition of more time. Within the single or choice cue, 4 types of cues were evaluated: initial consonant-vowel spoken, consonant-vowel written, rhyme, and repetition. The posttherapy analyses revealed a significant cor-

List of Abbreviations

$\begin{array}{ll}\text { ANOVA } & \text { analysis of variance } \\ \text { AoA } & \text { age of acquisition } \\ \text { BNT } & \text { Boston Naming Test } \\ \text { CVA } & \text { cerebrovascular accident } \\ \text { PALPA } & \begin{array}{c}\text { Psycholinguistic Assessment of Language } \\ \text { Processing in Aphasia }\end{array}\end{array}$


relation between the effect of facilitation across the 4 cueing conditions and overall therapy outcome for the group as a whole.

As well as providing prognostic indicators for anomia therapy, cueing has also been used as a technique to investigate the status of word knowledge in participants with differing aetiologies underlying their aphasia. For example, Jefferies and Lambon $\mathrm{Ralph}^{8}$ included phonologic cues in an array of tasks implemented to contrast the semantic impairment in participants with semantic dementia versus those with multimodal semantic impairment in the context of aphasia after stroke. While those with semantic dementia were not helped by phonemic cues, for participants with aphasia after stroke, picturenaming performance improved significantly with phonemic cues. ${ }^{9}$ The authors concluded that this contrast suggested those with aphasia after stroke retained semantic knowledge but have poor executive-semantic control, which is ameliorated by the external support afforded by cues. ${ }^{10,11}$ In semantic dementia, on the other hand, participants have a central degradation of semantic knowledge such that there is little activation in the speech production system, which can be boosted by phonemic cues.

A topic that is related to the effects of cueing in the aphasiology literature is what psycholinguistic factors/word properties influence accurate naming. In other words, the observation that certain groups of words prove to be cueable for a particular participant with aphasia and some are not cueable will relate to the underlying psycholinguistic properties of the words. A variety of psycholinguistic factors are likely to play a role in word retrieval, including word frequency, familiarity, length, age of acquisition (AoA), operativity, imageability, and concreteness. ${ }^{12,13}$ While word frequency has often been taken to be a particularly important variable in word retrieval, the intercorrelation of some measures has made it difficult to disentangle their specific contribution. ${ }^{14}$ In short, aphasic naming performance can be predicted by a combination of these factors (word frequency, AoA, imageability, and length). This begs the question of whether this can be extended to predict the cue length required to cue naming in aphasia- on the working hypothesis that words with positive psycholinguistic properties (eg, short, early acquired, high imageability) will have greater partial activation and will thus need less cueing to support naming.

This brief review of the literature regarding cueing in aphasiology has highlighted 2 themes. First, cueing has been used as a therapy technique, whereby repeated application can generate lasting improvements in later spontaneous naming. ${ }^{5,7}$ Second, cueing has been used as an indicator of current levels of word knowledge in participants with aphasia, whereby words which can be cued are closer to their activation threshold for accurate naming than those which are not. ${ }^{8,9}$ The present study combined both of these themes by investigating the process through which repeated and systematic cueing, as a therapy, improves word retrieval by tracing changes in response to cueing throughout therapy on a session-by-session basis. The specific research questions which this study addressed were: (1) Does the minimal level of cueing of specific words pretherapy predict their improved naming accuracy posttherapy? That is, are the items which are successfully named with minimal levels of cues those which will be most accurate at posttherapy assessments? Furthermore, if a relationship does exist between cue facilitation and therapy outcome, will this be maintained at the follow-up assessment point? (2) Which psycholinguistic factors predict which words are named most successfully after therapy? (3) Are the most cueable items also the ones that are reestablished at earlier points during therapy?

\section{METHODS}

We collated data from 3 previously published, case-series naming therapy studies that had been carried out by researchers within the Neuroscience and Aphasia Research Unit at the University of Manchester. ${ }^{15-17}$ These were articles which had used cueing hierarchies in treating anomic deficits in participants with aphasia. Participants, who were recruited from National Health Service Rehabilitation Services across the North West and West Midlands regions of England, had presented with chronic aphasia including word retrieval impairment. Identical inclusion criteria had been used across the 3 studies. All participants had suffered a left hemisphere cerebrovascular accident (CVA) at least 6 months prior to the start of the study. All were native speakers of English and literate prior to their CVA. People with additional medical conditions, for example, Parkinson's disease or dementia, were excluded from the studies. Furthermore, people with significant visual agnosia or verbal apraxia (as evidenced by very impaired performance on word to picture matching or word repetition tasks) were excluded, as severe deficits in these domains would have precluded their participation in therapy. Participants varied in their aphasia symptoms, severity, and time since CVA. Table 1 summarizes the participants' age, sex, handedness, occupation, months since CVA, and brief descriptions of their infarcts from computerized tomographic or magnetic resonance imaging scans (although this information was available for only 7 participants).

Table 2 shows the results of the background language assessments that the studies by Conroy, ${ }^{15,16}$ Snell ${ }^{17}$ and colleagues had in common. Table 3 shows the results of the cognitive assessments that the studies had in common.

\section{Baseline Performance on Language and Cognitive Assessment}

Each study had carried out language and cognitive assessments prior to treatment. The common assessments were extracted from each of these studies and are described briefly below. For all assessments, performance was judged to be impaired if the score fell 2 SDs below the mean or below the published cutoff score.

Naming. All participants had undergone pretreatment naming of the 60 items in the Boston Naming Test (BNT). ${ }^{18}$ The BNT contains 60 black and white line drawings of decreasing familiarity. This test was purely used as the measure of anomia severity (ie, without cueing). All participant results (see tables 2 and 3 ) are ordered by their BNT score.

Repetition. All participants had completed the Psycholinguistic Assessment of Language Processing in Aphasia $(\text { PALPA })^{19}$ subtest 9 , imageability $\times$ frequency word repetition, which contains 80 words of which 20 are highly imageable and high frequency, such as church and letter; 20 are highly imageable and low frequency, such as axe and tractor; 20 are low imageable and high frequency, such as attitude and thought; and 20 are low imageable and low frequency, such as dogma and mercy. A score below 78 was impaired.

Reading. All participants completed the PALPA ${ }^{19}$ subtest 31 , imageability $\times$ frequency, test of reading aloud. This test contains the same 80 words as PALPA 9. The normative cutoff score is 79 of 80 .

Semantic memory. All participants undertook the 3 picture version of the Pyramids and Palm Trees Test. ${ }^{20}$ This test assesses semantic knowledge by requiring participants to match 1 picture to another (from a choice of 2) on the basis of semantic association. For example, for a pyramid, the partici- 
Table 1: Descriptions of Participants

\begin{tabular}{|c|c|c|c|c|c|c|}
\hline Participant & Age $(y)$ & Sex & Handedness & Occupation & Months Postonset CVA & CT/MRI Description of Infarct \\
\hline$A$ & 75 & W & Right & Administrator & 50 & NA \\
\hline B & 42 & W & Right & Secretary & 46 & NA \\
\hline $\mathrm{C}$ & 74 & W & Right & NA & 72 & $\mathrm{~L}$ temporo-parietal \\
\hline $\mathrm{D}$ & 71 & M & Right & Farmer & 13 & NA \\
\hline$E$ & 75 & W & Right & Wages clerk & 13 & $\begin{array}{l}L \text { fronto-parietal extending into } \\
\text { the insular }\end{array}$ \\
\hline $\mathrm{F}$ & 48 & M & Right & Radio sports commentator & 16 & $\mathrm{~L}$ temporo-parietal \\
\hline G & 78 & W & Right & Accountant & 12 & NA \\
\hline $\mathrm{H}$ & 69 & M & Right & Metal worker & 55 & NA \\
\hline I & 60 & M & Right & Company director & 7 & NA \\
\hline$J$ & 84 & W & Right & Teacher & 10 & NA \\
\hline K & 58 & M & Right & Computer worker & 67 & $\mathrm{~L}$ fronto-parietal and insular \\
\hline $\mathrm{L}$ & 62 & W & Right & Housewife & 11 & NA \\
\hline M & 65 & W & Right & School secretary & 132 & NA \\
\hline $\mathrm{N}$ & 55 & M & Right & Railway maintenance & 7 & L parietal-temporal \\
\hline $\mathrm{O}$ & 48 & W & Right & Hairdresser & 136 & NA \\
\hline$P$ & 65 & M & Right & Engineer & 31 & NA \\
\hline $\mathrm{Q}$ & 62 & M & Right & Architect & 87 & NA \\
\hline $\mathrm{R}$ & 59 & M & Right & Bricklayer & 60 & NA \\
\hline $\mathrm{S}$ & 69 & M & Right & Processing manager & 11 & Subcortical \\
\hline $\mathrm{T}$ & 67 & M & Right & Lorry driver & 7 & $L$ frontal extending into insular \\
\hline$U$ & 65 & W & Right & Teacher & 56 & NA \\
\hline V & 53 & W & Right & Shop worker & 29 & NA \\
\hline
\end{tabular}

Abbreviations: CT, computed tomography; L, left; M, man; MRI, magnetic resonance imaging; NA, information not available; W, woman.

pant chooses between a palm tree and a fir tree. The published cutoff score for this test is 49 of 52 .

Attention. All participants were assessed on 2 auditory subtests of the Test of Everyday Attention. ${ }^{21}$ The first subtest (Elevator Counting) assesses sustained attention. Participants hear a series of beeps at random time intervals (representing floors in a lift). They are asked to count the number of beeps (range, 3-14) as if they were listening to an elevator lift climbing to a specific floor and then specify at which floor the lift had arrived. A table of written numbers was provided to enable responses from participants with number-naming difficulties. Use of their own fingers to indicate the number was

Table 2: Participants' Scores on Language Tests (in ascending order of naming)

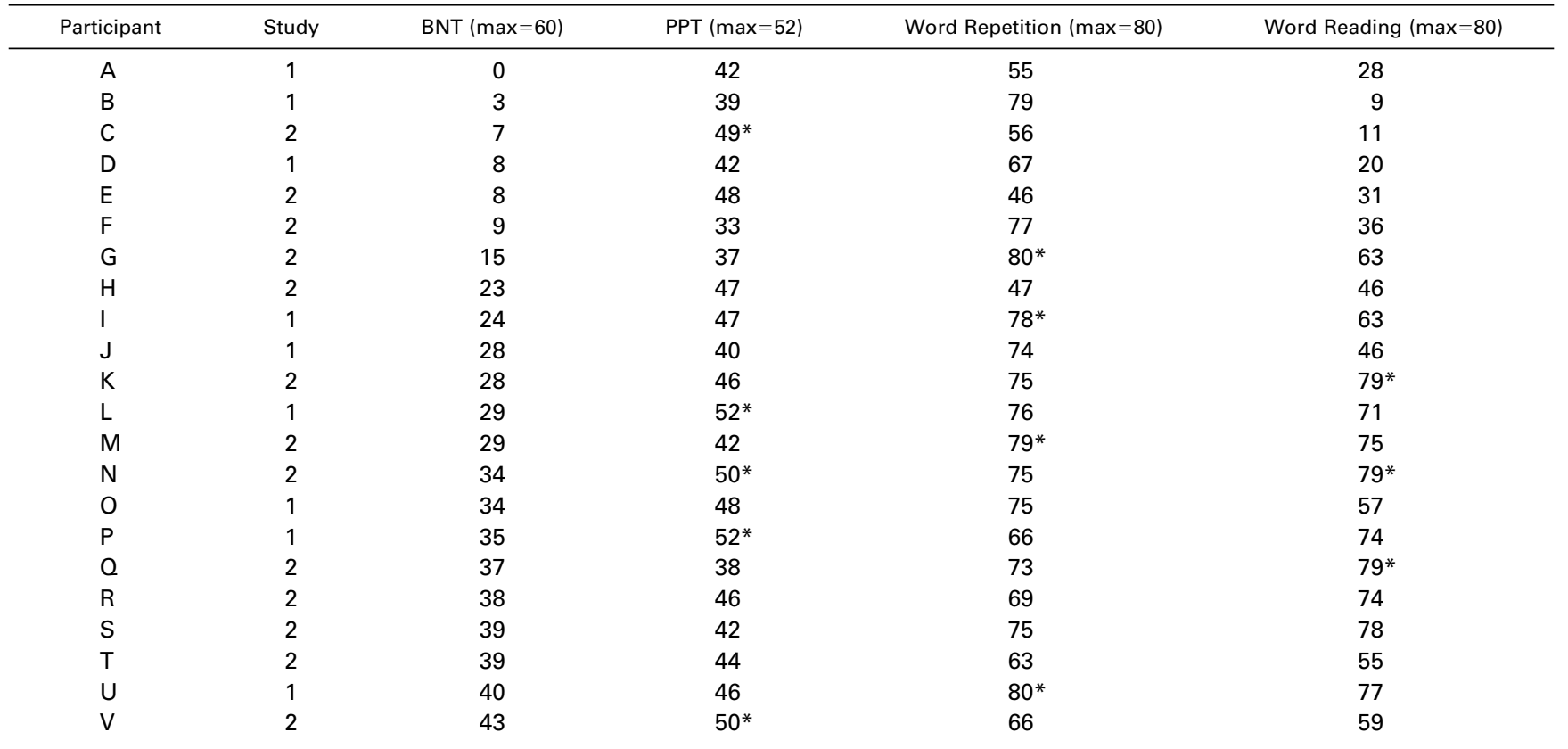

Abbreviations: PPT, Pyramids \& Palmtrees Test ${ }^{20}$; Study 1, Conroy et al ${ }^{15,16}$ studies; Study 2 , Snell et al ${ }^{17}$ study; Word Repetition: PALPA; Word Reading: PALPA. ${ }^{19}$

* Scores were within the normative range. 
Table 3: Participants' Scores on Cognitive Tests

\begin{tabular}{|c|c|c|c|c|c|c|c|}
\hline Participant & Study & TEA $(\max =7)$ & TEA-D $(\max =10)$ & WSCT $(\max =6)$ & Rey Copy $(\max =36)$ & Rey Imm (max=36) & Rey Delay $(\max =36)$ \\
\hline A & 1 & $7 *$ & 2 & $2 *$ & 23 & 3 & 6 \\
\hline B & 1 & 4 & 0 & $1 *$ & 26 & 5 & 7 \\
\hline $\mathrm{C}$ & 2 & $6^{*}$ & 1 & $5^{*}$ & $31 *$ & $17^{*}$ & $18 *$ \\
\hline $\mathrm{D}$ & 1 & $6^{*}$ & 1 & $2^{*}$ & $36^{*}$ & $11^{*}$ & 7 \\
\hline$E$ & 2 & $6 *$ & 4 & $3^{*}$ & 26 & 6.5 & 6.5 \\
\hline $\mathrm{F}$ & 2 & 4 & 0 & NA & 25 & 0 & 0 \\
\hline G & 2 & 3 & 1 & 0 & 19 & 0 & 0 \\
\hline $\mathrm{H}$ & 2 & $6^{*}$ & NA & $2^{*}$ & 15.5 & 4 & 3.5 \\
\hline I & 1 & 5 & $7 *$ & $5^{*}$ & $34^{*}$ & 11 & 11 \\
\hline $\mathrm{J}$ & 1 & 4 & 3 & $3 *$ & 18 & 6 & 4 \\
\hline $\mathrm{K}$ & 2 & $7 *$ & $6^{*}$ & $4^{*}$ & $32^{*}$ & $24^{*}$ & $21^{*}$ \\
\hline $\mathrm{L}$ & 1 & 5 & 1 & $1 *$ & $35^{*}$ & $22^{*}$ & $22^{*}$ \\
\hline $\mathrm{M}$ & 2 & $7 *$ & $6 *$ & $6 *$ & 22 & 2 & 1 \\
\hline $\mathrm{N}$ & 2 & $7 *$ & $5^{*}$ & $5^{*}$ & 32 & 6 & 5 \\
\hline 0 & 1 & $6 *$ & 4 & $4^{*}$ & $34^{*}$ & $19 *$ & $16^{*}$ \\
\hline Q & 2 & $7 *$ & 4 & $4^{*}$ & 27 & 19 & 15 \\
\hline $\mathrm{R}$ & 2 & $7 *$ & 2 & $2^{*}$ & 22 & $15^{*}$ & 11 \\
\hline $\mathrm{S}$ & 2 & $7 *$ & 2 & $2^{*}$ & 19 & 5 & 6.5 \\
\hline $\mathrm{T}$ & 2 & $7^{*}$ & $6^{*}$ & $6^{*}$ & NA & NA & NA \\
\hline$U$ & 1 & $7 *$ & 2 & $2^{*}$ & 28 & 7 & 7 \\
\hline V & 2 & $6^{*}$ & 4 & $4^{*}$ & $30 *$ & 11 & 5.5 \\
\hline
\end{tabular}

Abbreviations: NA, information not available; Rey Copy, Complex Figure of Rey-Copy trial22; Rey Delay, Complex Figure of Rey-Delayed recall $^{22}$; Rey Imm, Complex Figure of Rey-Immediate recall ${ }^{22}$; Study 1, Conroy et al ${ }^{15}, 16$ studies; Study 2 , Snell et al ${ }^{17}$ study; TEA, Test of Everyday Attention (elevator counting subtest) ${ }^{21}$; TEA/D, Test of Everyday Attention with distraction (elevator counting with distraction subtest) ${ }^{21}$; WCST, Wisconsin Card Sort Test. ${ }^{23}$

* Scores were within the normative range.

also accepted. Impaired performance on this test is 5 and below. The second subtest (Elevator Counting with Distraction) assesses divided attention. Participants hear a series of high and low beeps. They are asked to count only the low beeps, while ignoring the high beeps. Beeps are presented in a random order at random time intervals (range, 2-14). Impaired performance on this test is 4 and below.

Visuospatial memory. All participants carried out the full version of the Complex Figure of Rey. ${ }^{22}$ This test assesses participants' visuospatial ability by requiring them to copy a complex, abstract geometric figure. They are asked to reproduce it immediately from memory and again 30 minutes later, providing information about their visuospatial memory. Norms for this test are age-related.

Executive functioning. All participants were assessed with the Wisconsin Card Sort Test, ${ }^{23}$ which is a test of abstract reasoning/trial-and-error problem solving. Participants are required to match stimuli according to varying rules. We report the number of categories completed (maximum 6) to provide an indication of how many rule changes the participants were able to detect. Participants are impaired on this test if they fail to complete 1 category.

\section{Therapy Method}

A very similar therapy design and method was used in the 3 studies from which the data were collated. Target words were selected for therapy and control sets on the basis that participants had failed to name them on at least 2 of 3 presentations at baseline. Treatment and control sets were then matched for word frequency, number of phonemes, and baseline naming ability. The therapy method was essentially repeated picture naming of therapy targets aided by therapist-delivered progressive phonemic and orthographic cues (progressively longer cues were provided where necessary for each picture). Cueing continued until the word target was accurately produced; with maximal cueing, this would be repetition of the target word in spoken and written format. If the whole word was accurately produced via cueing then the participant was encouraged to repeat/read the target name. Each therapy consisted of 10 treatment sessions, followed by an assessment of naming accuracy for treated and control sets immediately after therapy (within 1wk) and 5 weeks later.

There were some differences between the studies by Conroy, ${ }^{15,16}$ Snell, ${ }^{17}$ and colleagues. Conroy investigated responsiveness to both noun and verb naming therapy. In order to match the Conroy and the Snell data, therefore, the verbs were removed and only nouns were included for the purposes of the present study. Furthermore, the studies diverged slightly with respect to the cueing hierarchy used and number of picture presentations during therapy. The Conroy studies used a cueing hierarchy, which consisted of a semantic cue (cue 1), then progressive phonemic and orthographic cues (cues 2-4). The semantic cue was implemented in order to allow for the possibility of errors being produced in naming (ie, it was predicted that it would be minimally helpful to participants in trying to name), because these studies were comparing errorful/trial and error methods with error-reducing/errorless methods (word repetition). The semantic cue did give, however, some support in naming, which may in part have related to offering more time to name, that is, greater latency for naming. In order to make the 3 studies comparable for analysis purposes, the phonemic cue data only was used.

Pretherapy naming accuracy across all the data was zero at baseline. In order to obtain a more fine-grained measure of pretherapy naming accuracy, we took the first picture presentation of items in the first therapy session and noted the minimal cue level required to facilitate accurate naming (given that cueing was progressive, that is, proceeding from minimal 
to maximal cues). This yielded 298 items from the Conroy studies and 782 items from the Snell study, giving a total of 1080 object naming targets. Within a large item-by-item analysis, for each individual item, we collated: (1) the initial cue proportion required to prompt accurate naming prior to therapy, (2) naming accuracy 1 week after the final therapy session (immediate posttherapy accuracy); and (3) naming accuracy 5 weeks later (follow-up assessment). The items were entered into the N-Watch software program, ${ }^{24}$ which provided psycholinguistic measures for most of the items, including word frequency, AoA, and imageability.

For the within-therapy cueing data, we narrowed the range of items in light of the high quantity of session-by-session cueing data this analysis entailed. Therefore, we collated the within therapy cue levels for the Conroy items only $(n=298)$. We took the cue level from the first of the 2 picture presentations per session, as the second picture presentation would more likely be open to the influence of a priming effect from the first picture presentation a few minutes previously. This provided us with 10 (cue level) data points for these 298 items during therapy.

\section{Method Summary}

The data analyzed in this study were extracted from 3 previously published studies. ${ }^{15,16,17}$ These studies were sufficiently similar in terms of participant recruitment, background language and cognitive testing, and therapy method, to allow for amalgamation of specific aspects of their data. Both baseline cueing data (the minimally effective cue as measured by the number of phonemes in the cue as a proportion of the target word) and postintervention accuracy data (including accurate and inaccurate words at an immediate and follow-up assessment point) were taken from all 3 studies. This provided 1080 words for statistical comparison of their baseline cue level and posttherapy accuracy, as well as comparison of the psycholinguistic values underpinning these 1080 words, that is, word frequency, imageability, and AoA. As a more focused and detailed analysis, we then took the 298 words, which had been used in 2 studies, ${ }^{15,16}$ and depicted how the baseline cue level changed through 10 therapy sessions, as part of a novel, withintherapy analysis.

\section{RESULTS}

The results are reported in order of the specific research questions posed in the Introduction.

(1) Does the minimal level of cueing of specific words predict their improved naming accuracy posttherapy? That is, are the items which are successfully named with minimal levels of cues those which will be most accurate at posttherapy assessments? Furthermore, if a relationship does exist between cue facilitation and therapy outcome, will this be maintained up to the follow-up assessment point?

In order to answer these questions, we calculated the successful cue for spontaneous naming prior to the onset of therapy and, in order to equate across items of different length, we expressed this as a proportion of the target word length (eg, target word comb (3 phonemes); cue used/k/(1 phoneme); cue proportion $=.33$ ) for all 1080 items. We then analyzed this cue proportion in relation to both the immediate and follow-up posttherapy accuracy results.

The cue proportions required for items which were correctly named at the immediate posttherapy assessment were shorter than those used for items which were not named posttherapy (mean cue proportion for correct items $\pm \mathrm{SD}, .53 \pm .27$; mean cue for incorrect items $\pm \mathrm{SD}, .61 \pm .28 ; t=4.42 ; \mathrm{df}=1077$; $P<.001,2$-tailed). The pattern also held when the cueing data were split by accuracy at the follow-up assessment. Again, the pretherapy cue was shorter for the successfully named items at follow-up assessment than the unnamed items (mean cue for correct items $\pm \mathrm{SD}, .51 \pm .27$; mean cue for incorrect items \pm $\mathrm{SD}, .63 \pm .28 ; t=7.02 ; \mathrm{df}=1077 ; P<.001,2$-tailed). In order to account for severity and other participant-related differences, we reanalyzed the same data using a logistic regression analysis where therapy outcome for each item (either immediate or follow-up) was simultaneously predicted from the level of cueing required prior to therapy and participant as a categorical predictor variable. This replicated the previous analysis in that both cue level (immediate posttherapy testing: Wald $=9.90$, $P=.002$; follow-up testing: Wald $=33.8, P<.001)$ and participant (immediate posttherapy testing: Wald $=136.4, P<.001$; follow-up testing: Wald $=146.2, P<.001$ ) were significant independent predictors of item therapy outcome.

These results indicated that a shorter cue in the pretherapy assessment was an indicator that the item would be named accurately in both posttherapy assessments. Conversely, the items which required more substantial cues were less likely to be named accurately posttherapy. These relationships were maintained between the immediate and follow-up assessment points.

(2) Which psycholinguistic factors predict which words are named most successfully after therapy?

A logistic regression analysis was performed with the required cue (as a proportion of the word length) prior to therapy as the dependent variable and celex frequency, imageability, and word AoA as predictor variables. A significant model emerged $\left(F_{3,463}=2.902, P=.035\right)$. Celex frequency and AoA were not significant predictors (celex frequency: $\beta=.03, P>.50$; AoA: $\beta=.02, P>.60)$, but imageability was a significant predictor $(\beta=-.124, P=.018)$. Celex frequency and AoA were highly significantly correlated (Spearman's $\rho=-.607, \quad P<.001$, 2-tailed).

Considering the results obtained for the first 2 research questions, it is evident that there was a significantly predictive 3-way relationship between cue level, posttherapy accuracy, and word imageability. In other words, highly imageable words tended to require lower cue levels at the start of therapy and these were the words which were more likely to be named accurately after therapy. This pattern also held for the follow-up assessment point.

(3) Are the most cueable items also the ones that are reestablished at earlier points during therapy?

We analysed the cross-therapy session changes in the required cue by stratifying the data according to the posttherapy accuracy measures. Here, we considered 3 different outcome categories: (1) consistently correct (ie, correct at both immediate and follow-up assessment points), (2) inconsistently correct (ie, correct at 1 assessment point and incorrect at the other), and (3) consistently incorrect (ie, not named at either assessment point). Figure 1 shows the mean cue level across the 10 therapy sessions for these 3 groups of items.

We analyzed these data using a 2-way analysis of variance (ANOVA) with the factors time (ie, across the 10 therapy sessions) and posttherapy accuracy grouping (ie, correct, incorrect, or inconsistent). There was a significant effect of time $\left(F_{9,2673}=131.74, P<.001\right)$, a significant effect of accuracy grouping $\left(F_{2,297}=107.79, P<.001\right)$, and a significant interaction between time and accuracy grouping $\left(\mathrm{F}_{18,2673}=3.422, P<.001\right)$.

However, as can be inferred from figure 1, there appeared to be a strong change in required cue level during sessions 1 through 5, which then stabilized across sessions 6 through 10 . 


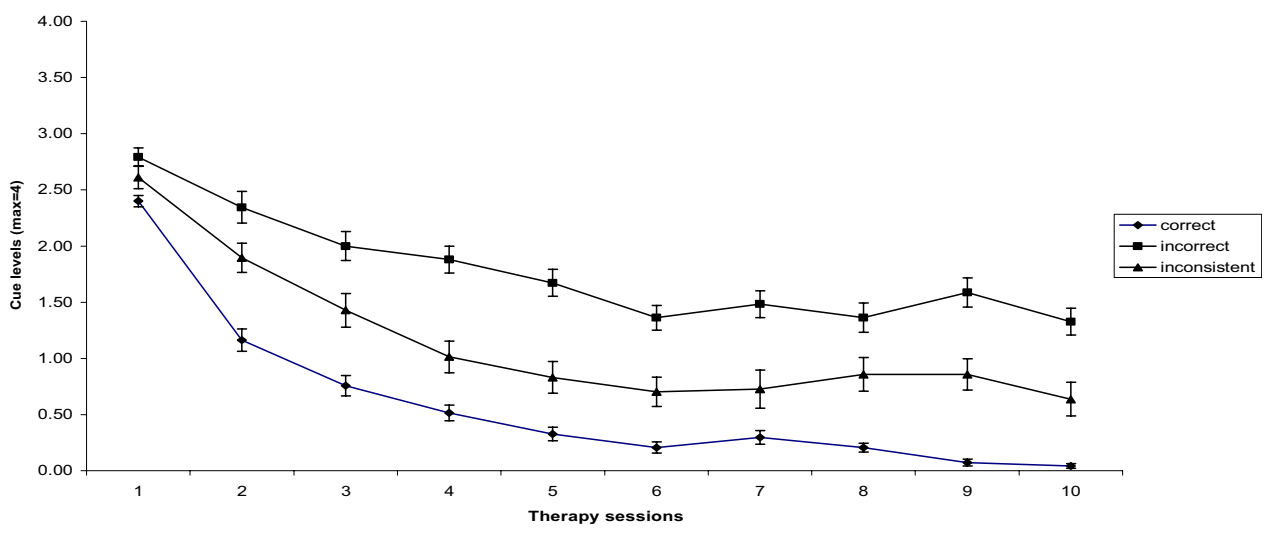

Fig 1. Mean cue level required during therapy for items named correctly versus inconsistently versus unnamed posttherapy. Correct, incorrect, and inconsistent in the legend refer to the posttherapy naming accuracy for these items (ie, whether they were correct on both assessments, incorrect on both, or correct on 1 only). The cue levels on the $y$ axis depict the cue hierarchy, where $0=$ no cue, $1=$ semantic cue, $\mathbf{2}=$ minimal phonemic and graphemic cue, $3=$ more substantial phonemic and graphemic cue, and $4=$ whole word presented in phonemic and graphemic form. The error bars represent the SE.
This suggested that the significant effect of time may have derived purely from the changes which occurred in the first 5 therapy sessions. In order to test this hypothesis, we conducted separate analyses of the effects of time and accuracy grouping during sessions 1 through 5 and sessions 6 through 10. To simplify the interpretation of each ANOVA, we compared the results for the first and last sessions in each dataset (ie, first vs fifth session, sixth vs tenth session only). From session 1 to 5 , there was a significant effect of time $\left(F_{1,297}=590.99, P<.001\right)$, a significant effect of accuracy grouping $\left(F_{2,297}=38.625\right.$, $P<.001)$, and a significant interaction between time and accuracy $\left(F_{2,297}=16.477, P<.001\right)$. During session 6 to 10 , there was no effect of time $\left(F_{1,297}=1.86, P=.17\right)$, a significant effect of accuracy grouping $\left(F_{2,297}=82.919, P<.001\right)$, and no interaction between time and accuracy $\left(F_{2,297}<1\right)$. As can be seen in figure 1 , the interaction between time and accuracy grouping in the first half of the session data is due to the fact that the required cue drops more for items that are named posttherapy (both consistently or inconsistently), but that the drop for the unnamed items posttherapy is smaller.

\section{DISCUSSION}

This study was concerned with the question of whether it is possible to predict therapy outcome for individual items by using cueing data. In particular, we investigated whether therapy outcome could be predicted from the level of cueing required to prompt correct naming pretherapy and the psycholinguistic properties of these words. We also assessed the evolution of this relationship across the 10 sessions of therapy. Analyzing a large corpus of words taken from studies based on 22 participants with chronic aphasia, we found that there was a statistically significant relationship between words which were successfully named with relatively minimal cueing prior to the start of therapy and words named without support posttherapy. Hence, the words which were most readily cued tended to be the ones which were most likely to be accurately named after therapy. Conversely, the words, which required more substantial cues at the start of therapy, were less likely to be named after therapy.

In many ways, this initial finding is unsurprising. There is a certain degree of circularity to the contention that the items that are easier at baseline are then easier to improve on in therapy. However, cueing has been seen as a light, clinical tool, which is suggestive rather than definitive, as a prognostic indicator for word retrieval. These findings, however, which were based on a large dataset of words across many participants, have the benefit of showing the relationship between cueing and improvability to be a robust one. There are at least 2 more compelling issues to consider after the observation that cueable words turn out to be easier to improve on in therapy.
First, there are implications of this initial finding for rehabilitation. The cognitive neuropsychologic therapy literature, which has been characterized by this microlevel analysis of item responsiveness in aphasia therapy, has typically found item-specific improvement in word retrieval studies (see Whitworth et $\mathrm{al}^{25}$ for an overview). In other words, the elusive finding of generalized improvement in word retrieval skills, for example, as indicated by greater accuracy scores on treated and untreated sets of target words, has been the exception rather than the rule in published studies. ${ }^{26}$ This has led to several studies in which the primary goal has been improved reliability of production for purposefully trained items, for example, usually vocabulary items, which are agreed to be communicatively relevant to an individual participant, ${ }^{5,27-29}$ where generalized improvement in word retrieval skills was taken to be a possible but not a probable therapy outcome. Such studies have negotiated target words for training on the basis of likely everyday use in conversation or general interaction; proper names of family members and friends are one example. ${ }^{29}$ Successful minimal cueing could constructively serve as a screening tool within this therapy approach, alongside personal communicative relevance, in terms of determining items likely or not likely to respond to naming therapy.

Therefore, the extent to which a corpus of words assessed for baseline (preintervention) naming accuracy is easily cueable, as a readily included method within confrontation naming would be predictive of likely success in naming therapy for an individual participant with aphasia. Furthermore, successful minimal cueing may provide a more personalized screen, relative to considering psycholinguistic variables, of likely naming success in therapy, in that it may well relate to underlying psycholinguistic factors (ie, highly imageable and frequent words which were acquired early in childhood, generally stand the best chance for improving with therapy) but equally it may well not. In other words, measures such as word frequency and word imageability are derived with reference to large samples of respondents. For particular individuals with aphasia, certain words which most of us would consider infrequent and of low imageability may well be neither, as a consequence of high personal relevance and salient experience. For example, a participant with aphasia who is very engaged by communication technology may find terms referring to systems for information transfer (eg, WiFi) more mentally imageable as concepts compared with others with (or indeed without) aphasia. Cueing as a screening tool for likely naming success, therefore, has a role here beyond its associations with psycholinguistic variables.

The second significant issue to emerge from the initial finding of the robust relationship between ready cueability and 
improvement in naming accuracy through therapy is the psycholinguistic factor which underpins this relationship. We found that there was a significant influence of imageability on ease of cueing (the most concrete words required the smallest cue) but no evidence for an influence of AoA or frequency. This was a striking finding given that, because all the studies had used object pictures, there was a very restricted range of imageability values. In this regard it is useful to note that previous studies that have explored the relationship with picture naming accuracy in aphasic datasets have also found imageability to be a significant predictor. For example, Nickels and Howard ${ }^{12}$ found that imageability predicted spontaneous naming performance and they attributed this finding to the richness of semantic representations for a particular word, rather than how visually imageable a specific picture is per se. The current study extends the same finding in that it suggests both spontaneous naming and therapy outcome can be predicted by the semantic richness of the word. A key question is why might this be the case? There are at least 2 possibilities that could be tackled in future studies. The first is that the participants included in this study, like many participants with aphasia related to middle cerebral artery strokes, present with significant phonologic impairment but variable semantic deficits. There is now clear evidence that there is a productive interaction between semantic and phonologic representations such that the phonologic activation and integrity of word forms is provided in part by input from the semantic system. ${ }^{30,31}$ As such, if some words have intrinsically stronger underlying semantic representations then they may be more likely to be named and also respond better to therapy. Second, evidence from nonstroke clinical groups and functional neuroimaging in neurologically intact participants suggests that extra-sylvian regions (especially the basal anterior temporal lobe) play a critical role in semantic representation. ${ }^{32,33}$ This region sits outside the supply of the middle cerebral artery and thus is typically intact in most participants with stroke-related aphasia. Putting these factors together might suggest that the intrinsic strength of the semantic representations in this basal anterior temporal lobe region and their interaction with any remaining peri-sylvian language regions might be a critical predictor in spontaneous language ability and responsiveness to therapy.

A further important and clinically significant finding was that the relationship between minimal cueing and posttherapy accuracy could be seen very clearly in the within-therapy session data. Although the required cue reduced during therapy for all targets (as hoped and expected), there was a clear difference across the different items depending on their eventual naming accuracy posttherapy (named, inconsistently named, unnamed). Throughout therapy, the items with the best therapy outcome always required the shortest cue, and the drop-in cue length was greatest for these items across sessions. By session 6 , the change across therapy sessions was minimal and the cueing data flat-lined from that point. This begs some important questions about how much therapy may be required to arrive at the naming success achieved after session 10 . Although it might be tempting to conclude that fewer (eg, only 5) rather than more $(\mathrm{eg}, 10)$ sessions were required, there are reasons to be cautious. For example, although cueing/accuracy stabilized at session 6 , it is possible that this period may act as an important consolidation phase for the learning from sessions 1 through 5. Indeed it is possible that, although accuracy did not change, the efficiency of naming might improve during this second phase and help to preserve the therapy effect at the immediate and follow-up phases. To have a definitive answer, future studies will need to directly compare therapy outcome for, for example, 5 versus 10 sessions of therapy, both in terms of immediate and longer-term therapy effects.

\section{Study Limitations}

As a retrospective meta-analysis of previously implemented studies, this study did have limitations with regard to the compatibility of the 3 studies in question. Specifically, there were some differences in collation of demographic details (information from brain scans), as well as slightly varying cueing hierarchies. Although, these factors make some aspects of interpretation of the data less straightforward than would ideally have been the case, we believe they do not detract from the robustness of the data and the findings.

\section{CONCLUSIONS}

This study has demonstrated a significantly predictive 3-way relationship between shorter phonemic cues at baseline, naming accuracy, and word imageability; highly imageable words required less cueing and were more likely to be accurately named posttherapy. A novel analysis of cross-session accuracy revealed that, even though the required cue length reduced across the first 6 of 10 therapy sessions, the relationship between required cue length and final posttherapy accuracy was present throughout therapy. Further research should be directed toward understanding the cognitive underpinnings of this learning, in terms of the nature of the interactions between semantic and phonologic representations as depleted systems. Also, further research could usefully focus on dosage issues with regard to such learning, such as the amount of training required for consolidation in the learning process for immediate and, crucially, longer term effects.

Acknowledgement: We would also like to thank Professor David Howard, $\mathrm{PhD}$, for helpful guidance at the inception of the project.

References
1. Basso A, Capitane I, VignoIol A. Influence of rehabilitation of language skills in aphasic patients: a controlled study. Arch Neurol 1979;36:190-6.

2. Schuell H, Jenkins JJ, Jiminez-Pabon E. Aphasia in adults. New York: Harper \& Row; 1964.

3. Marshall RC, Neuburger SI, Phillips DS. Effects of facilitation and cueing on labelling of «novel $\gg$ stimuli by aphasic subjects. Aphasiology 1992;6:567-83.

4. Howard D, Patterson K, Franklin S, Orchard-Lisle V, Morton J. Treatment of word retrieval deficits in aphasia. Brain 1985;108: $817-29$.

5. Hickin J, Best W, Herbert R et al. Phonological therapy for word-finding difficulties: a re-evaluation. Aphasiology 2002;16: 981-99.

6. Howard D. Cognitive neuropsychology and aphasia therapy: the case of word retrieval. In: Papathanasiou I, editor and translator. Acquired neurogenic communication disorders. London: Whurr Publishers; 2000.

7. Marshall J. Aphasia therapy and cognitive neuropsychology: a promise still to be fulfilled? In: Joffe V, Cruice M, Chiat S, editors and translators. Language disorders in children and adults. Chichester: Wiley-Blackwell; 2008.

8. Jefferies E, Lambon Ralph MA. Semantic impairment in stroke aphasia vs. semantic dementia: a case-series comparison. Brain 2006;129:2132-47.

9. Jefferies E, Patterson K, Hopper S, et al. Deficits of knowledge vs. executive control in semantic cognition: insights from cued naming. Neuropsychologia 2008;46:649-58.

10. Corbett F, Jefferies E, Ehsan S, Lambon Ralph MA. Different impairments of semantic cognition in semantic dementia and 
semantic aphasia: evidence from the non-verbal domain. Brain 2009;132:2593-608.

11. Noonan KA, Jefferies E, Corbett F, Lambon Ralph MA. Elucidating the nature of deregulated semantic cognition in semantic aphasia: evidence for the roles of prefrontal and temporoparietal cortices. J Cogn Neurosci 2010;22:1597-613.

12. Nickels L, Howard D. Aphasic naming: what matters? Neuropsychologia 1995;33:1281-303.

13. Ellis A, Lum C, Lambon Ralph M. On the use of regression techniques for the analysis of single case aphasic data. J Neurolinguistics 1996;9:165-74.

14. Cuetos F, Monsalve A, Perez A. Determinants of lexical access in anomia. J Neurolinguistics 2005;18:383-99.

15. Conroy P, Sage K, Lambon Ralph MA. Errorless and errorful therapy for verb and noun naming in aphasia. Aphasiology 2009; 23:1311-37.

16. Conroy P, Sage K, Lambon Ralph MA. The effects of decreasing and increasing cues for verb and noun naming in aphasia. Aphasiology 2009;23:707-30.

17. Snell C, Sage K, Lambon Ralph MA. How many words should we provide in anomia therapy? A meta-analysis and a case series study. Aphasiology 2010;24:1064-94.

18. Goodglass H, Kaplan E, Barresi B. The Boston Diagnostic Aphasia Examination. 3rd ed. Philadelphia: Lippincott, Williams \& Wilkins; 2001.

19. Kay J, Lesser R, Coltheart M. Psycholinguistic Assessments of Language Processing in Aphasia (PALPA). Hove: Lawrence Erlbaum Associates; 1992.

20. Howard D, Patterson K. The Pyramids and Palm Trees Test: a test of semantic access from words and pictures. Bury St Edmunds: Thames Valley Test Co; 1992.

21. Robertson IH, Ward T, Ridgeway V, Nimmo-Smith I. The Test of Everyday Attention (TEA). Bury St Edmunds: Thames Valley Test Co; 1994.

22. Meyers JE, Meyers KR. Rey Complex Figure Test and Recognition Trial. Odessa: Psychological Assessment Resources; 1995.
23. Grant DA, Berg EA. Wisconsin Card Sorting Test. San Antonio: Psychological Assessment Resources; 1993.

24. Davis CJ. N-Watch: A program for deriving neighbourhood size and other psycholinguistic statistics. Behavior Research Methods 2005;37:65-70.

25. Whitworth A, Webster J, Howard D. A Cognitive neuropsychological approach to assessment and intervention in aphasia: a clinician's guide. Hove: Psychology Pr; 2005.

26. Nickels L. Therapy for naming disorders: revisiting, revising and reviewing. Aphasiology 2002;16:935-79.

27. Best W, Greenwood A, Grassly J, Hickin J. Bridging the gap: can impairment-based therapy for anomia have an impact at the psycho-social level? Int J Lang Commun Disord 2008;43:390407.

28. Herbert R, Best W, Hickin J, Howard D, Osborne F. Combining lexical and interactional approaches to therapy for word finding deficits in aphasia. Aphasiology 2003;17:1163-86.

29. Robson J, Marshall J, Pring T et al. Processing proper nouns in aphasia: evidence from assessment and therapy. Aphasiology 2004;18:917-35.

30. Patterson K, Lambon Ralph MA, Jefferies E, et al. "Pre-semantic" cognition in semantic dementia: six deficits in search of an explanation. J Cogn Neurosci 2006;18:169-83.

31. Jefferies E, Crisp J, Lambon Ralph MA. The impact of phonological or semantic impairment on delayed auditory repetition: evidence from stroke aphasia and semantic dementia. Aphasiology 2006;20:963-92.

32. Binney RJ, Embleton KV, Jefferies E, Parker GJ, Ralph MA. The ventral and inferolateral aspects of the anterior temporal lobe are crucial in semantic memory: evidence from a novel direct comparison of distortion-corrected fMRI, rTMS, and semantic dementia. Cerebral Cortex 2010;20:2728-38.

33. Sharp DJ, Scott SK, Wise RJ. Retrieving meaning after temporal lobe infarction: the role of the basal language area. Ann Neurol 2004;56:836-46. 